\title{
User collaborated reception of spatially multiplexed signals: An experimental study in group mobility
}

\section{AUTHOR(S):}

Shubhi, Ilmiawan; Hayashi, Yuji; Murata, Hidekazu

\section{CITATION:}

Shubhi, IImiawan ... [et al]. User collaborated reception of spatially multiplexed signals: An experimental study in group mobility. IEICE Transactions on Fundamentals of Electronics, Communications and Computer Sciences 2017, E100.A(1):227-231

\section{ISSUE DATE:}

2017-01-01

URL:

http://hdl.handle.net/2433/261147

\section{RIGHT:}

(C) 2017 The Institute of Electronics, Information and Communication Engineers; 許諾条件に基づいて掲載しています。 


\title{
User Collaborated Reception of Spatially Multiplexed Signals: An Experimental Study in Group Mobility
}

\author{
Ilmiawan SHUBHI ${ }^{\dagger a)}$, Student Member, Yuji HAYASHI ${ }^{\dagger}$, Nonmember, and Hidekazu MURATA ${ }^{\dagger}$, Senior Member
}

\begin{abstract}
SUMMARY In multi user multiple input multiple output systems, spatial precoding is typically employed as an interference cancellation technique. This technique, however, requires accurate channel state information at the transmitter and limits the mobility of the mobile station (MS). Instead of spatial precoding, this letter implements collaborative interference cancellation (CIC) for interference suppression. In CIC, neighboring MSs share their received signals without decoding and equivalently increase the number of received antennas. The performance is evaluated through a field experiment using a vehicle that is equipped with seven MSs and moves around an urban area.

key words: collaborative interference cancellation, field experiments, $M U$ MIMO, spatial multiplexing
\end{abstract}

\section{Introduction}

Considering its potential to improve the spectral efficiency, multi-user multiple-input multiple-output (MUMIMO) systems have been attracted many researchers in wireless communication areas [1], [2]. Through MUMIMO, the advantages of MIMO systems can be preserved even when the number of received antennas in the mobile station (MS) is limited. Owing to the distance between the MSs, MU-MIMO ensures that the channel correlation is relatively low and preserves the spatial multiplexing gain [3].

To mitigate inter-user interference, MU-MIMO typically employs spatial precoding techniques such as those in [4] and [5]. These techniques, however, require accurate downlink channel state information (CSI) at the transmitter. Unfortunately, accurate CSI is hard to obtain, especially when the MSs have a relatively high velocity [6]. Therefore, MU-MIMO with spatial precoding techniques primarily considers low-mobility scenarios [7]-[10].

Instead of spatial precoding, the interference cancellation capability of the MS can be improved by sharing received signals to virtually increase the number of received antennas. In this letter, we label this technique collaborative interference cancellation (CIC) [11]. This technique does not require accurate CSI at the transmitter and is suitable for high user density and high mobility environments such as when the users are on trains and busses.

The performance of CIC in an actual environment, however, depends on several factors. Unlike the theoretical scenario which mostly consider i.i.d. Rayleigh fading,

Manuscript received April 15, 2016.

Manuscript revised August 19, 2016.

${ }^{\dagger}$ The authors are with the Graduate School of Informatics, Kyoto University, Kyoto-shi, 606-8501 Japan.

a)E-mail: ilmiawan@dco.cce.i.kyoto-u.ac.jp DOI: 10.1587/transfun.E100.A.227 actual environments experiencing channel shadowing and channel correlation between MSs. In the signal processing at the MSs, the differences at the MS sampling time, frequency offset, and signal to noise ratio might also effect the CIC performance. In addition, the reliability of the interuser links in a mobile environment, also need to be confirmed.

Therefore, we conduct this field experiment to confirm the effectiveness of MU-MIMO with CIC in an actual environment. In our field experiment, a BS with multiple transmit antennas is employed to serve several active MSs. These active MSs are located in a car that moves at a certain speed. In addition to the four active MSs, other MSs are also available in the car and can be used in CIC. Therefore, the number of collaborating MSs is larger than or equal to that of the active MSs. These collaborating MSs then conduct CIC by sharing their received signals through short range wireless communication links. Afterward, the maximum likelihood detection (MLD) and minimum mean square error (MMSE) are used as the detection algorithms for obtaining the transmitted data.

At the cost of higher computational complexity, MLD will outperform MMSE in terms of bit error rate (BER) performance. It should be noted, however, the number of available MSs in trains or busses will most likely be higher than that of active MSs. In that sense, we can increase the number of collaborating MSs to be greater than the number of active MSs and employ a simple linear detection algorithm such as MMSE. The large number of collaborating users can also be exploited to combat non ideal condition which occurs in the actual environment, e.g. the channel correlation among MSs.

Part of this work had been presented in [12]-[14]. In this letter, more complete results and analysis are given. The performance comparison of MLD and MMSE for MUMIMO with CIC in actual environments is presented for the case of equal theoretical diversity order. Another result on the reliability of the interuser links is also plotted. The received power and the BER vs. packet index for all MSs are shown. In addition, a more complete cumulative distribution function $(\mathrm{CDF})$ of the BER is given. Furthermore, the effect of the moving speed is also described.

\section{MU-MIMO with CIC}

Figure 1 shows the system model considered in the field experiment. A BS with $M$ transmit antennas serves $L$ active MSs out of $N$ collaborating MSs. The transmission be- 


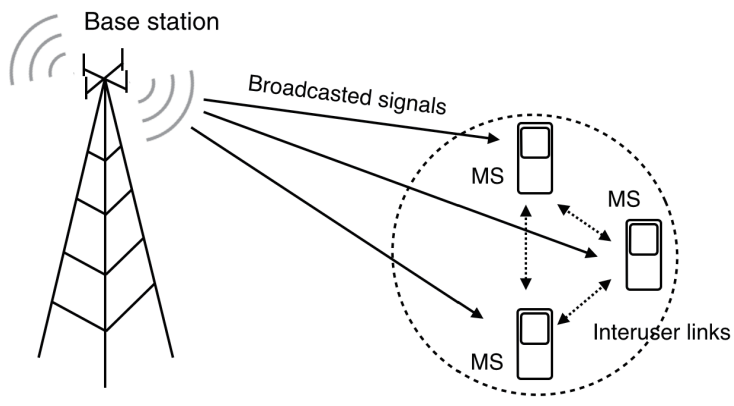

Fig. 1 MU-MIMO with collaborative interference cancellation.

tween BS and MSs occupies the same frequency and time resources to create an MU-MIMO scenario. In this letter, we consider the case where $L=M$ and each MS is equipped with one receive antenna.

First, $M$ transmit antennas in the BS transmit $M$ independent streams. In this experiment, the raw BER performance, that is, the BER performance for the case of no channel code is employed, is investigated. On the receiver side, $N$ MSs conduct CIC by sharing their received signals and equivalently increase the number of their received antennas. Let $\boldsymbol{y}=\left[y_{1} y_{2} \ldots y_{N}\right]^{\mathrm{T}}$ where $y_{n}$ is the received signal for $n$th MS. The received signals for $N$ collaborating MSs can be written as

$$
\boldsymbol{y}=\boldsymbol{H} \boldsymbol{x}+\boldsymbol{n}
$$

where $\boldsymbol{H} \in \mathbb{C}^{N \times M}$ is an $N \times M$ matrix of a wireless channel, $\boldsymbol{x}$ is an $M \times 1$ vector of transmitted symbols, and $\boldsymbol{n}$ is an $N \times 1$ vector of noise.

In this field experiment, the performance of MUMIMO with CIC is observed for both MLD and MMSE algorithms. By using MLD, the vector of transmitted symbols can be obtained by

$$
\hat{\boldsymbol{x}}=\underset{\boldsymbol{x} \in X}{\operatorname{argmin}}\|\boldsymbol{y}-\boldsymbol{H} \boldsymbol{x}\|^{2}
$$

where $\mathcal{X}$ is the set of all possible vectors of transmitted symbols. For the case of MMSE, $\hat{\boldsymbol{x}}$ are obtained by exploiting the correlation of the received signals as

$$
\hat{\boldsymbol{x}}=\boldsymbol{H}^{\mathrm{H}} \boldsymbol{R}_{y y}^{-1} \boldsymbol{y}
$$

where (.) $)^{\mathrm{H}}$ is the Hermitian transpose of (.) and $\boldsymbol{R}_{y y}$ is the correlation matrix of the received signal vector including the data and the cyclic redundancy check (CRC).

\section{Experiment Setup}

Table 1 shows the parameters used in this experiment. Figure 2(a) shows signal generators that generate four quadrature phase shift keying (QPSK) signals at a symbol rate of $312.5 \mathrm{k}$ symbols/s. These QPSK signals are then sent to four antennas in the BS. The height of the BS is $25.5 \mathrm{~m}$. In the BS, the antennas are installed in perpendicular positions, as shown in Fig. 2(b). From the BS, packet signals
Table 1 Experiment parameters.

\begin{tabular}{l|r}
\hline System Parameters & Values \\
\hline Number of antennas of BS & 4 \\
Number of antennas of MS & 1 \\
Number of active MSs & 4 \\
Number of collaborating MSs & 4,7 \\
Frame length & $50 \mathrm{~ms}$ \\
Carrier frequency & $5.11 \mathrm{GHz}$ \\
Symbol rate & QPSK \\
Modulation & Values \\
\hline BS Parameters & $312.5 \mathrm{k} \mathrm{symbols/s}$ \\
\hline Transmit filter & Square Root Nyquist \\
& (roll-off factor=0.4) \\
Antenna hight & $25.5 \mathrm{~m}$ \\
Antenna gain & $5 \mathrm{dBi}$ \\
\hline MS Parameters & Values \\
\hline Antenna gain & $3 \mathrm{dBi}$ \\
ADC resolution & 14 bits \\
\hline Packet Configuration & Values \\
\hline Synchronization word & 15 BPSK symbols \\
Training sequence & 16 BPSK symbols \\
Control & 15 QPSK symbols \\
Data + CRC & 80 QPSK symbols \\
\hline
\end{tabular}

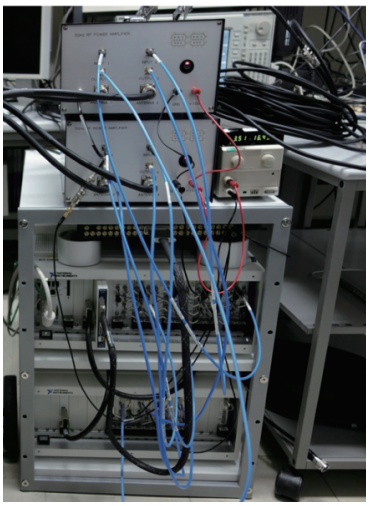

(a) Signal generators.

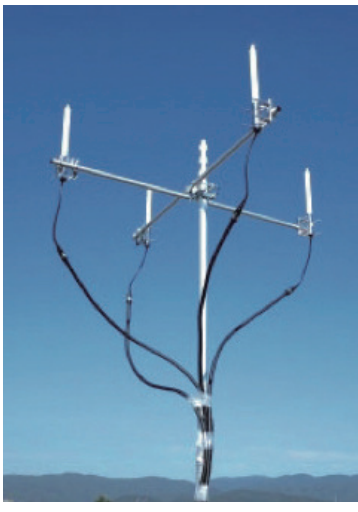

(b) Antennas.
Fig. 2 Transmitter setup.

are broadcasted to the MSs. Each of these packets contains 15 binary phase shift keying (BPSK) symbols of synchronization word, 16 BPSK symbols of training sequence, 15 QPSK symbols for control, and 80 QPSK symbols of data, including the CRC.

The BS in this experiment is located at a building in Kyoto University. The MSs are installed inside a car that moves along the route shown in Fig. 3. The route can be divided into two parts. In part $\mathrm{A}$, the speed of the car is about $15 \mathrm{~km} /$ hour, and in part $\mathrm{B}$, the speed of the car is about $45 \mathrm{~km} /$ hour. Inside the car, seven universal software radio peripherals (USRPs) are used to represent the MSs. Each USRP in Fig. 4(a) uses $3 \mathrm{dBi}$ antenna gains. Inside the USRP, an analog to digital converter (ADC) with 14 bit resolution is used. The position of each MS can be seen in Fig. 4(b). To conduct a signal exchange, WiFi connections with IEEE $802.11 \mathrm{n}$ in the $5 \mathrm{GHz}$ band are used as interMS communications. Each MS broadcasts its quantizedreceived-signal to the other MSs in a dedicated time slot by 


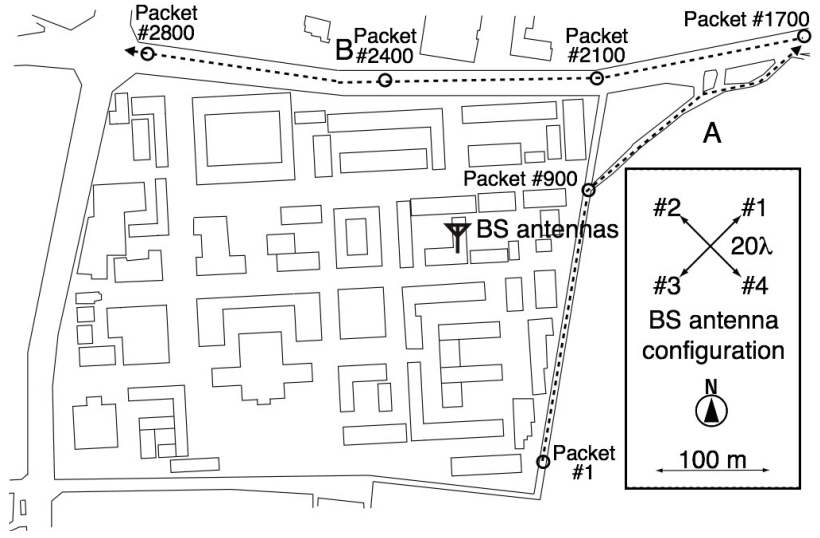

Fig. 3 Experiment route.

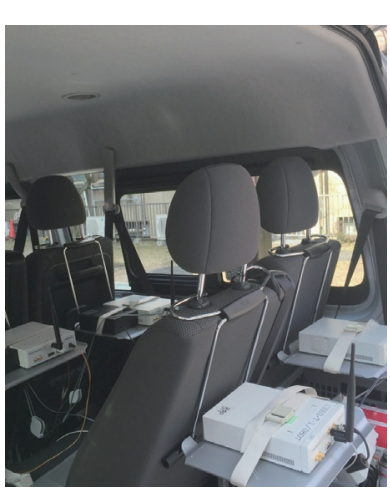

(a) USRP + PC.

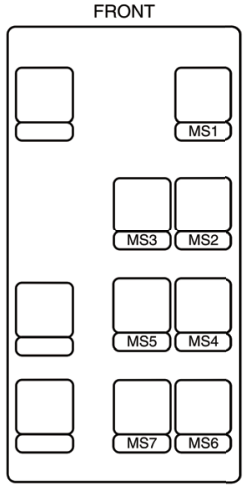

(b) MS positions.
Fig. 4 MS setup.

using User Datagram Protocol (UDP). For each I and Q part, 8 bits quantization is used.

\section{Experiment Results}

The results obtained through our field experiment can be seen in Figs. 5-8. In Figs. 5-7, packet index from 1 to 1700 corresponds to the part $\mathrm{A}$ of the route, while packet index from 1701 to 2800 corresponds to the part B of the route. Figure 5 shows the number of signals successfully shared to MS1 for each packet of transmitted data. The average numbers of signals successfully shared to MS2, MS3, and MS4 are also shown. From this figure, we can observe that the interuser links for signal information exchange are mostly successful and very reliable.

In Fig. 6, the received power of the transmitted signals of the BS at MS1 is shown. The average power at MS1 is $-95 \mathrm{dBm}$. The median of the received power at all active MSs is also shown. From the figure, it can be seen that all of the MSs have similar received powers. This similar received power, together with an equal number of received signals from the other MSs, results in a similar BER performance of all MSs for all packets, as shown in Fig. 7. In this figure, the black-dashed line is the BER for the case of $4 \times 4$ MLD, while the red line is the BER for the case of $4 \times 7$ MMSE.

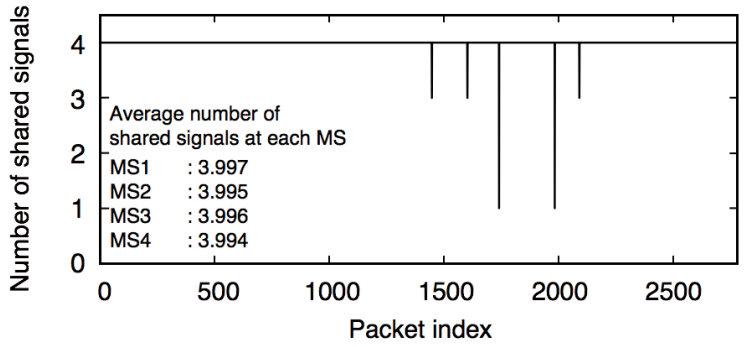

(a) Four collaborating MSs

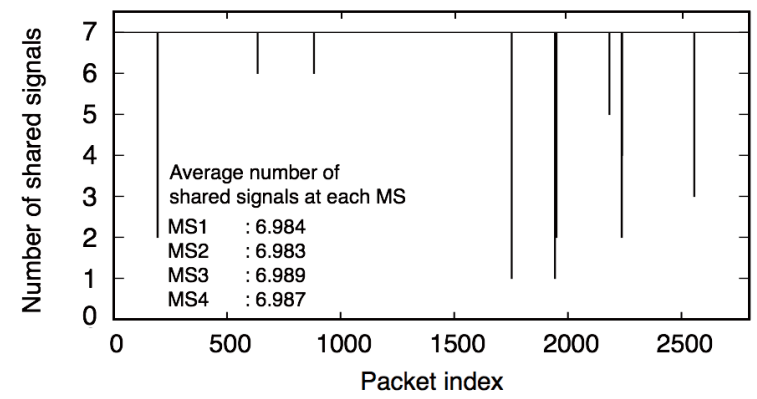

(b) Seven collaborating MSs.

Fig. 5 Number of shared signals at MS1.

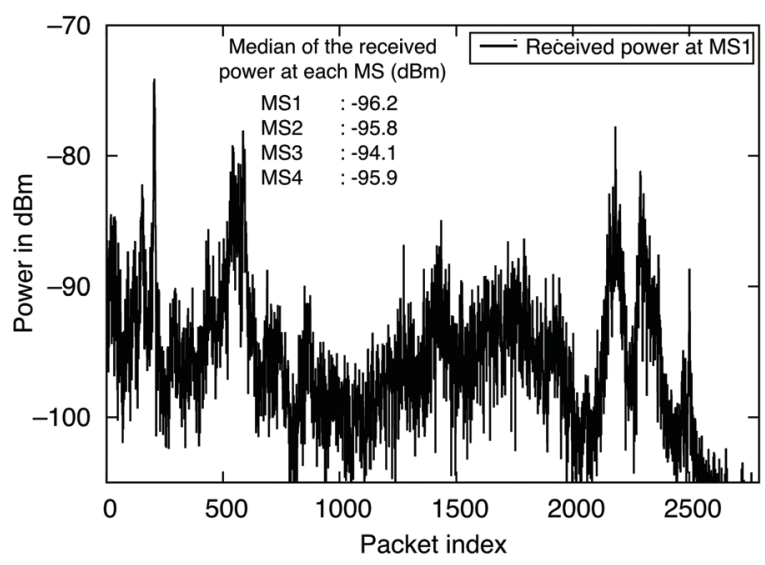

Fig. 6 Received power measured at MS1.

Next, the CDF of the BER of MU-MIMO with CIC is shown in Fig. 8 for the case of $N=4$ using MLD and for the case of $N=7$ using both MLD and MMSE. In the CDF graph, the instantaneous BER is calculated for every 10 packets of data. As shown in the figure, the BER performance at the part A is better than the BER performance at the part $\mathrm{B}$. This circumstance occurs as the received power in the part A is larger than that of part B due to the difference on the geographical topology. In addition, the average speed in the part B is higher, causing the channel response fluctuates faster. In addition, it can be seen from the figure that at the cost of higher complexity, MLD obtains better performance compared to that of MMSE when an equal value of $N$ is used. However, it can also be seen that by having more array gain, $4 \times 7$ MMSE performs better than $4 \times 4$ 

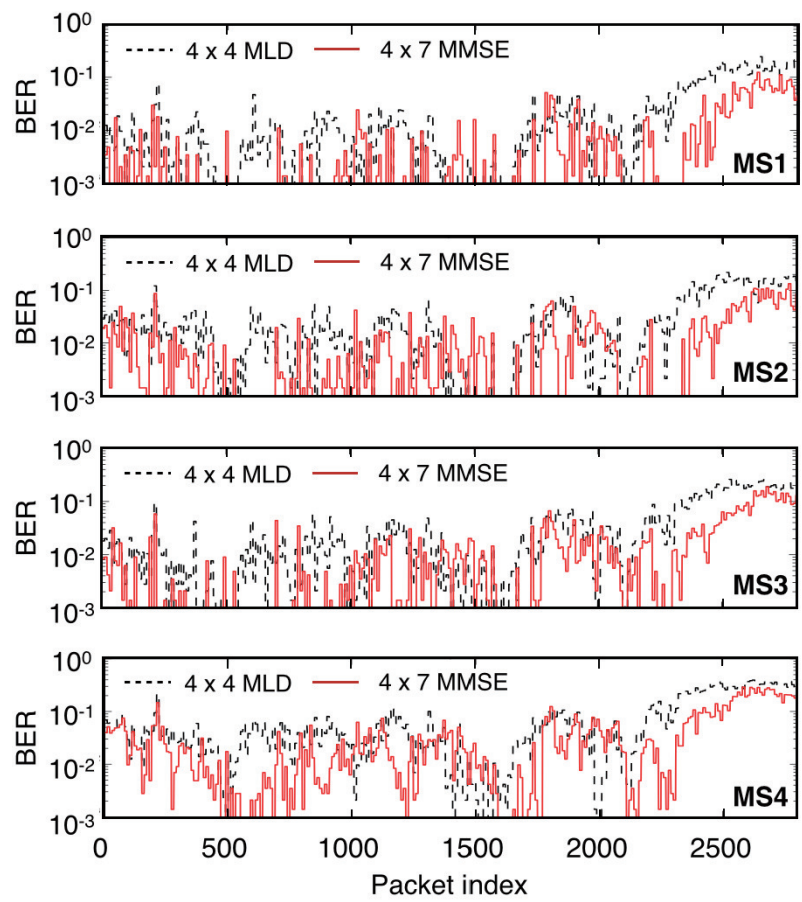

Fig. 7 BER in each packet transmission.

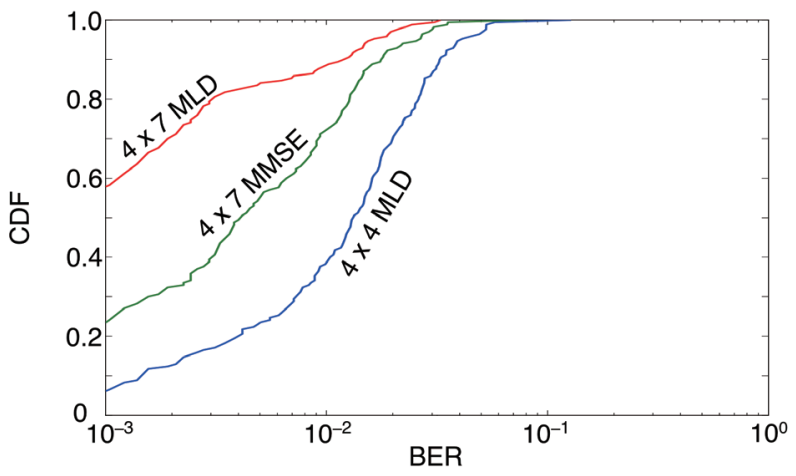

(a) Part A of the route $(15 \mathrm{~km} / \mathrm{hour})$.

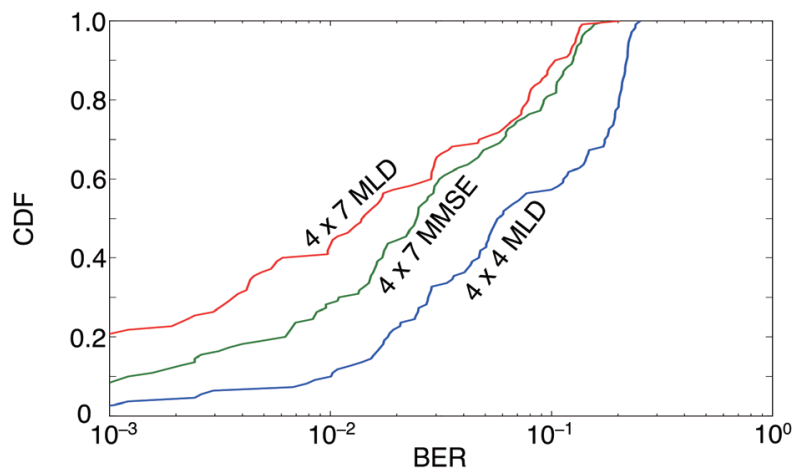

(b) Part B of the route $(45 \mathrm{~km} / \mathrm{hour})$

Fig. 8 CDF of BER of MU-MIMO with CIC

MLD although these scenarios have a similar theoretical diversity. This better performance is obtained for both cases of average speed.

\section{Conclusion}

This letter presented field experiment results of MU-MIMO communications which employ CIC as the interference cancellation method. In CIC, collaborating MSs share their received signals and increase the number of their received antennas. Our field experiments show that in the actual environment, MU-MIMO with CIC is applicable, despite of the moving nature of the group mobility. As accurate CSI at the transmitter is not required, CIC could become a potential approach for MU-MIMO communications in group mobility scenarios. In addition, it is also shown that in this field experiment, a higher number of collaborating users with MMSE has better performance compared to that of smaller number of collaborating users with MLD even when the theoretical diversity order is equal.

\section{Acknowledgment}

This work was supported by Strategic Information and Communications R\&D Promotion Programme (SCOPE) of the Ministry of Internal Affairs and Communications, Japan. This research is partly supported by the joint research project with NTT DoCoMo.

\section{References}

[1] F. Boccardi, B. Clerckx, A. Ghosh, E. Hardouin, G. Jöngren, K. Kusume, E. Onggosanusi, and Y. Tang, "Multiple-antenna techniques in LTE-advanced," IEEE Commun. Mag., vol.50, no.3, pp.114-121, March 2012.

[2] C. Lim, T. Yoo, B. Clerckx, B. Lee, and B. Shim, "Recent trend of multiuser MIMO in LTE-advanced," IEEE Commun. Mag., vol.51, no.3, pp.127-135, March 2013.

[3] Q.H. Spencer, C.B. Peel, A.L. Swindlehurst, and M. Haardt, "An introduction to the multi-user MIMO downlink," IEEE Commun. Mag., vol.42, no.10, pp.60-67, Oct. 2004.

[4] M. Joham, W. Utschick, and J.A. Nossek, "Linear transmit processing in MIMO communications systems," IEEE Trans. Signal Process., vol.53, no.8, pp.2700-2712, Aug. 2005.

[5] K. Zu, R.C. de Lamare, and M. Haardt, "Multi-branch TomlinsonHarashima precoding design for MU-MIMO systems: Theory and algorithms," IEEE Trans. Commun., vol.62, no.3, pp.939-951, March 2014.

[6] J. Zhang, M. Kountouris, J. Andrews, and R. Heath, "Multi-mode transmission for the MIMO broadcast channel with imperfect channel state information," IEEE Trans. Commun., vol.59, no.3, pp.803814, March 2011.

[7] Y. Inoue, K. Saito, T. Kawamura, and H. Andoh, "Field experiments on antenna configuration associated with fading correlation for downlink multi-user MIMO in LTE-advanced," Proc. IEEE 77th Vehicular Technology Conference (VTC Spring), pp.1-5, 2013.

[8] Y. Hatakawa, T. Matsumoto, K. Kitagawa, and S. Konishi, "Indoor experiment of 8-by-2 multiuser MIMO transmission using Tomlinson-Harashima-precoding subject to limited CSI feedback," Proc. IEEE Radio and Wireless Symposium (RWS), pp.49-51, 2014.

[9] D. Takeda, Y. Inoue, K. Saito, T. Kawamura, and H. Andoh, "Field experiments on antenna element grouping for smart vertical MIMO in LTE-advanced downlink," Proc. IEEE 80th Vehicular Technology Conference (VTC2014-Fall), pp.1-5, 2014. 
[10] G. Dahman, J. Flordelis, and F. Tufvesson, "Experimental evaluation of the effect of BS antenna inter-element spacing on MU-MIMO separation," Proc. IEEE International Conference on Communications (ICC), pp.1685-1690, 2015.

[11] H. Mao, W. Feng, and N. Ge, "MIMO broadcast channels with cooperation among densely clustered receivers," Proc. IEEE International Conference on Communication Systems, pp.288-292, 2014.

[12] H. Murata and R. Shinohara, "Performance improvement of ZFprecoded MU-MIMO transmission by collaborative interference cancellation," IEICE Commun. Express, vol.4, no.5, pp.155-160, May 2015.
[13] Y. Hayashi and H. Murata, "Field experimental evaluation of collaborative interference cancellation with linear and non-linear signal processing schemes," IEICE Trans. Commun. (Japanese Edition), vol.J98-B, no.7, pp.689-695, July 2015.

[14] Y. Hayashi, I. Shubhi, and H. Murata, "User collaboration for interference cancellation on multi-user MIMO communication systems," Proc. IEEE 82nd Vehicular Technology Conference (VTC2015Fall), pp.1-5, 2015. 